\title{
Fähigkeitsausweis Stethoskopie (SAAM)
}

\author{
Ryan Tandjung ${ }^{a}$, Hans Rudolf Koelz ${ }^{b}$, Werner Bauer ${ }^{c}$

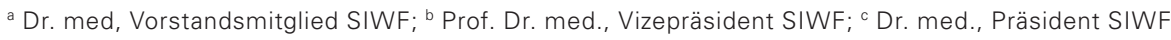

\section{Vorbemerkung}

Früher wurden neue Facharzttitel und Fähigkeitsausweise in der Schweizerischen Ärztezeitung vorgestellt. Das SIWF möchte diese Tradition wiederbeleben und stellt den seit dem 1. April 2015 neu gültigen Fähigkeitsausweis Stethoskopie vor. Die Wichtigkeit der Aus- und Weiterbildung der Basiskenntnisse in der körperlichen Untersuchung kann nicht genügend betont werden. In der Anerkennung des Engagements der Swiss Academy of Auscultatory Medicine hat deshalb der SIWF-Vorstand der Schaffung dieses Fähigkeitsausweises zugestimmt. Dieser tritt ab 1. April 2015 in Kraft. Eine Verknüpfung des Fähigkeitsausweises mit einer TARMEDPosition ist in Verhandlung.

\section{Einleitung}

Die heute sehr technisch orientierte Medizin lässt die Grundfähigkeiten der klinischen Untersuchung oft ausser Acht. Die Kunst der Auskultation geht deshalb zunehmend verloren. Daher wurde die Swiss Academy of Auscultatory Medicine (SAAM) gegründet, die der Auskultation wieder stärker ihren alten Stellenwert geben soll. Die Schaffung des Fähigkeitsausweis Stethoskopie trägt zur Anerkennung der Bedeutung der klinischen Untersuchung am Krankenbett bei.

\section{Certificat d'aptitude}

Le certificat d'aptitide de stéthoscopie de la Swiss Academy of Auscultatory Medicine (SAAM) sera introduit le $1^{\text {er }}$ avril 2015. Ce nouveau certificat d'aptitude a été créé pour tenir compte de l'importance de la transmission des bases dans l'examen clinique. La condition requise pour passer le certificat d'aptitude est un titre de spécialiste en médecine interne générale, en pédiatrie ou en chirurgie. L'obtention du certificat nécessite deux ans d'activité au sein d'un établissement de formation postgraduée reconnu, la participation à quatre modules de deux jours chacun (cours de base, cœur et poumons, abdomen et grands vaisseaux, cours final), ainsi que la réussite de l'examen final. Le coût du certificat d'aptitude s'élève à $998 \mathrm{CHF}$ (TVA en sus). Dans le cadre des dispositions de transition, les médecins qui ont exercé une activité clinique pendant au moins 10 ans et qui s'enregistrent auprès de la SAAM par le biais de l'adresse Internet www.saez.ch/fa stethoskopie d'ici le 30 avril 2015 peuvent bénéficier de conditions assouplies pour l'obtention du certificat.

\section{Voraussetzungen}

Voraussetzung für den Erwerb des Fähigkeitsausweises ist ein Facharzt für Allgemeine Innere Medizin, Kinder- und Jugendmedizin oder Chirurgie. Ebenso eine mindestens zweijährige Tätigkeit an einer anerkannten Weiterbildungsstätte, sowie der Besuch von insgesamt vier zweitägigen Kursmodulen. Das Ziel der Weiterbildung ist es, die theoretischen Grundsätze eng mit der praktischen Anwendung zu verknüpfen.

Das erste Modul setzt sich mit den Grundlagen der Akustik und der Membranphysiologie sowie dem Aufbau von Stethoskopen auseinander. Modul 2 widmet sich theoretisch und praktisch der Auskultation von Lunge und Herz. Modul 3 der Auskultation des Abdomens und der grossen Gefässe, sowie der Diagnose von Gaseinschlüssen in Muskulatur und Bindegewebe. Abschlusskurs ist Modul 4. Die Zulassung hierzu setzt den Nachweis von 500 Auskultationen von Herz und der Lunge voraus (je 250 davon superauskultiert durch einen zertifizierten Tutor). Dazu müssen 150 abdominelle Auskultationen nachgewiesen werden.

Die Schlussprüfung erfolgt nach Abschluss der vier Module. Die Evaluation umfasst eine Multiple ChoicePrüfung für den theoretischen Background und einen Hörverständnistest unter Laborbedingungen. In Zentrum der anschliessenden praktischen Prüfung stehen die Handhabung der Trage- und Grifftechniken und der Umgang mit Patienten während der Auskultation anhand von 50 klinischen Fällen im Zentrum.

Die Gebühren für die Module werden durch die SAAM festgelegt. Für die Erteilung des Fähigkeitsausweises wurde ein Betrag von 998 CHF (+ MwSt) festgelegt.

\section{Übergangsbestimmung}

Im Rahmen einer Übergangsbestimmung können Ärztinnen und Ärzte, die mehr als zehn Jahre klinische Tätigkeit nachweisen, den Fähigkeitsausweis zu erleichterten Bedingungen erhalten. Unter der Voraussetzung der regelmässigen aktiven Auskultationstätigkeit, der Erfüllung der Grundvoraussetzungen, sowie einer Registration bei der SAAM via Internetadresse www.saez. ch/fa_stethoskopie wird bis 30. April 2015 der Fähigkeitsausweis ohne weitere Formalitäten vergeben. 